\title{
Parametric CFD Analysis to Study the Influence of Fin Geometry on the Performance of a Fin and Tube Heat Exchanger
}

\author{
Shobhana Singh, Kim Sørensen, Thomas J. Condra \\ Department of Energy Technology, Pontoppidanstræde 9220, Aalborg East, Denmark, \\ ssi@et.aau.dk; kso@et.aau.dk; tc@et.aau.dk
}

\begin{abstract}
Heat transfer and pressure loss characteristics of a fin and tube heat exchanger are numerically investigated based on parametric fin geometry. The cross-flow type heat exchanger with circular tubes and rectangular fin profile is selected as a reference design. The fin geometry is varied using a design aspect ratio as a variable parameter in a range of $0.1-1.0$ to predict the impact on overall performance of the heat exchanger. In this paper, geometric profiles with a constant thickness of fin base are studied. Three-dimensional, steady-state CFD model is developed using commercially available Multiphysics software COMSOL v5.2. The numerical results are obtained for Reynolds number in a range from 5000 to 13000 and verified with the experimentally developed correlations. Dimensionless performance parameters such as Nusselt number, Euler number, efficiency index, and area-goodness factor are determined. The best performed geometric fin profile based on the higher heat transfer and lower pressure loss is predicted. The study provides insights into the impact of fin geometry on the heat transfer performance that help escalate the understanding of heat exchanger designing and manufacturing at a minimum cost.
\end{abstract}

Keywords: fin and tube heat exchanger, numerical modelling, fin profile, conjugate heat transfer, turbulent flow, pressure loss

\section{Introduction}

Fins are the extended surfaces used in heat exchangers to enhance the heat transfer rate between heat transfer surfaces and the flowing fluid (Cengel et al., 2012). The increment in the heat transfer performance through fin surfaces is widely employed in many industrial applications. Application of waste heat recovery systems has received tremendous attention during the last decade due to the resulting saving of primary fuel, increased energy efficiency and lower greenhouse gas emissions. Heat exchangers are one of the important components of these waste heat recovery systems. During past few years, H-type finned and tube heat exchangers have been studied both experimentally ( $\mathrm{Yu}$ et al., 2010; Chen and Lai, 2012; Chen et al., 2014) and numerically (Tong, 2007; Zhang et al., 2010; Jin et al., 2013). The studies mainly focused on examining the heat transfer and flow resistance characteristics for a reference design of the H-type finned tube bundles. In addition, combined heat and mass transfer analysis on $\mathrm{H}$-type design with three types of finned tube namelydimple finned tube, longitudinal vortex generators (LVGs) finned tube, and finned tube with compound dimples and LVGs together was conducted (Wang and Tang, 2014; Zhao et al.,2014).

The implementation of fins on the primary heat surface enhances the complexity, volume, and weight which make the design and construction of fin surfaces of vital importance in heat exchanger applications. Very limited research on different fin types or geometry profiles is available due to restricted experimental conditions and numerical challenges. This limitation overshadows the current knowledge of design factors that influence the heat transfer and pressure loss characteristics. Hence, it becomes imperative to study the different fin geometric profiles to determine the optimal fin design for a given H-type fin and tube heat exchanger application.

In this paper, we used Computational Fluid Dynamics (CFD) to obtain the solution of governing equations of physical phenomena in a cross-flow type fin and tube heat exchanger. The parametric study of fin geometry is conducted using air as a working fluid considering the 'rectangular' fin as reference geometric profile. Heat transfer and pressure loss characteristics in a fin and tube heat exchanger with different geometric fin profiles are predicted and compared with the reference fin profile geometry.

\section{Numerical model development}

\subsection{Heat exchanger design}

The heat exchanger used in the present study is fin and tube type. The design entails circular tubes and rectangular fins which are attached to the set of two tubes with a fixed gap in between. This particular design is also called 'H-type' finned tube heat exchanger due to the typical arrangement of fins on tubes resembling the letter ' $\mathrm{H}$ '. An orderly arrangement of the single units 


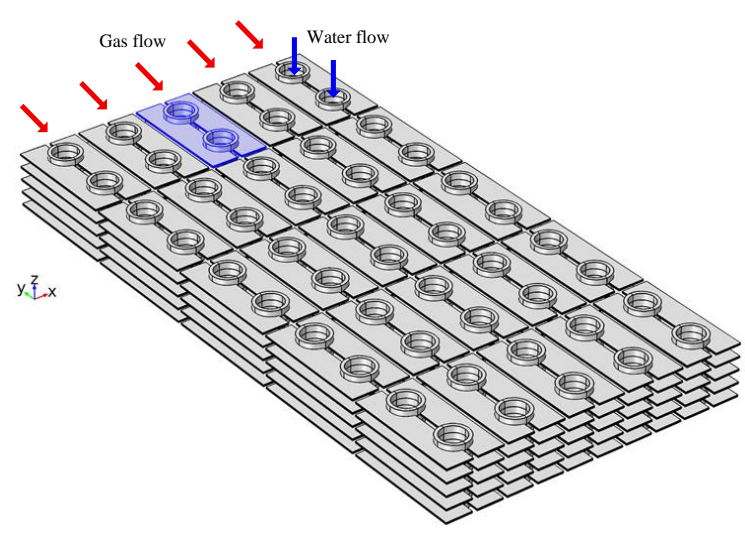

Figure 1. Double fin and tube (or H-type) heat exchanger configuration.

results in the complete heat exchanger configuration which can be scaled for desired applications based on the heat transfer rate and allowable pressure loss. Figure 1 shows the pictorial view of fin and tube heat exchanger configuration used in the present study. The design typically used in waste heat recovery applications such as marine boilers, where hot exhaust gas flows over the finned tube bundle, and cold water flows inside the tubes as can be seen in Figure 1. The heat transfers from hot exhaust gases, by convection through fins and conduction within fin and tube thickness, to the water inside the tubes for steam generation for other application purposes

\subsection{Computational geometry}

The geometry of the fin and tube heat exchanger simulated in the present study is shown in Figure 2. In order to save the computational effort, the geometry to be studied is reduced to one-half of the single unit. The computational geometry is divided into three domainsfin, tube and gas; and boundaries- inlet, outlet, and symmetry. The geometric dimensions of the heat exchanger design are given in Table 1.

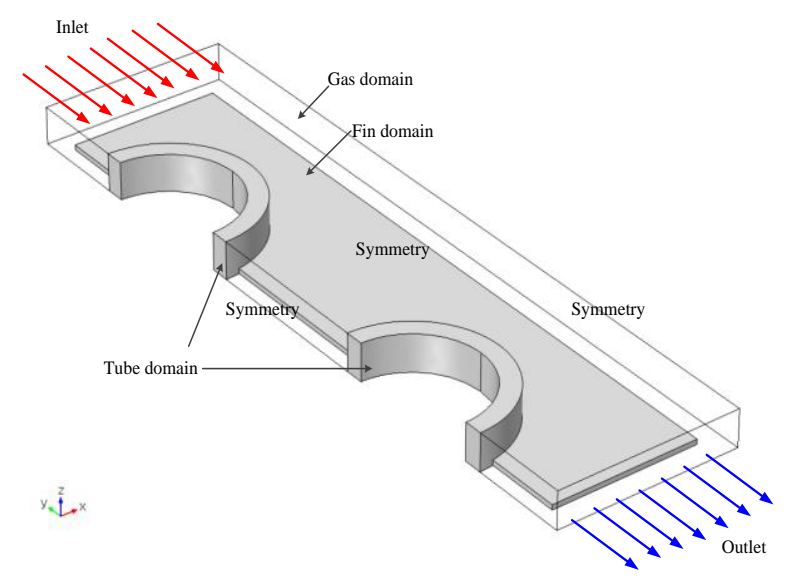

Figure 2. Computational geometry used in the present investigation.
Table 1. Design parameters and operating conditions for a single unit of the exchanger.

\begin{tabular}{|l|l|l|l|}
\hline Parameter & Symbol & Value & Unit \\
\hline Length of the fin & $L_{f}$ & 0.145 & $\mathrm{~m}$ \\
\hline Width of the fin & $W_{f}$ & 0.070 & $\mathrm{~m}$ \\
\hline $\begin{array}{l}\text { Thickness of the reference } \\
\text { fin base }\end{array}$ & $\delta_{f b, r}$ & 0.002 & $\mathrm{~m}$ \\
\hline $\begin{array}{l}\text { Thickness of the reference } \\
\text { fin tip }\end{array}$ & $\delta_{f t, r}$ & 0.002 & $\mathrm{~m}$ \\
\hline $\begin{array}{l}\text { Width of the gap between } \\
\text { fins }\end{array}$ & $\delta_{a}$ & 0.007 & $\mathrm{~m}$ \\
\hline Inner diameter of the tube & $D_{i}$ & 0.030 & $\mathrm{~m}$ \\
\hline Outer diameter of the tube & $D_{o}$ & 0.038 & $\mathrm{~m}$ \\
\hline Tube pitch & $p_{t}$ & 0.077 & $\mathrm{~m}$ \\
\hline Length of the gas domain & $L_{g}$ & 0.155 & $\mathrm{~m}$ \\
\hline Width of the gas domain & $W_{g}$ & 0.080 & $\mathrm{~m}$ \\
\hline Fin pitch & $p_{f}$ & 0.015 & $\mathrm{~m}$ \\
\hline Temperature at gas inlet & $T_{i n}$ & 573.15 & $\mathrm{~K}$ \\
\hline Pressure at gas outlet & $p_{\text {out }}$ & 0.0 & $\mathrm{~Pa}$ \\
\hline $\begin{array}{l}\text { Temperature of inner tube } \\
\text { wall }\end{array}$ & $T_{w}$ & 453.15 & $\mathrm{~K}$ \\
\hline
\end{tabular}

\subsection{Formulation of the fin geometric profile}

In the present work, the geometry of the fin is varied using aspect ratio $(\alpha)$ as a profile parameter which is defined as the ratio of thickness of fin tip $\left(\delta_{f t}\right)$ to the thickness of fin base $\left(\delta_{f b}\right)$ and can be expressed as-

$$
\alpha=\frac{\delta_{f t}}{\delta_{f b, r}}
$$

The rectangular geometry of the fin is considered as a reference profile to simplify the analysis and geometric complexity, and the thickness of fin base is kept constant as of reference rectangular fin $\left(\delta_{f b, r}\right)$ while the thickness of the fin tip is subjected to a variation (Figure 3). The aspect ratio is varied in a range $\alpha=1.0$ 0.1 transforming the reference rectangular fin profile (at $\alpha=1.0$ ) into the trapezoidal profile (at $\alpha=0.7,0.5,0.3$ ) which eventually resembles a triangular profile (at $\alpha=0.1$ ). With the change in aspect ratio, total heat transfer area, the thermal contact area between the fin and tubes and, the weight of the heat exchanger unit (computational geometry) changes as shown in Figure 4. 

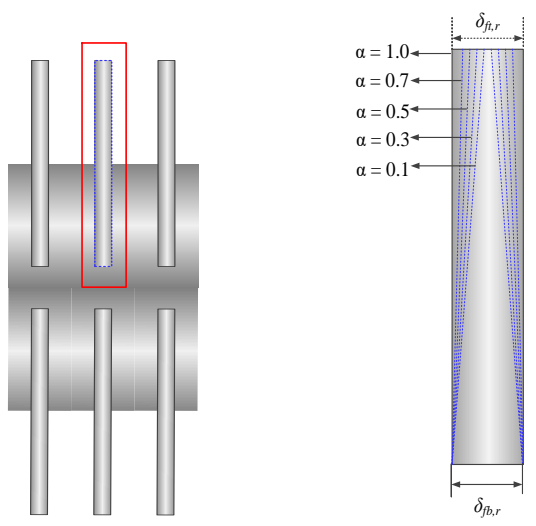

Figure 3. Computational geometry used in the present investigation.

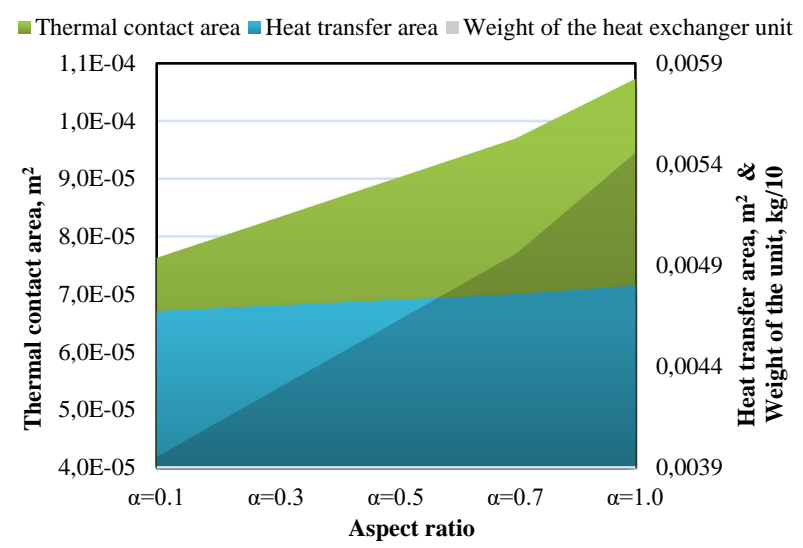

Figure 4. Computational geometry used in the present investigation.

\subsection{Governing equations}

3D CFD model is developed using commercially available Multiphysics software COMSOL v5.2. Following assumptions are made in the present model-

- Steady state flow and heat transfer

- Incompressible flow

- Negligible thermal contact resistance

- Temperature dependent fluid property

- Constant inner tube wall temperature

- No periodic boundary condition (i.e. model is valid for the first unit of the heat exchanger as shown in Figure1).

The mass and momentum balance for flow in the gas domain and energy balance in terms of heat transfer are given as-

$$
\begin{gathered}
\nabla \cdot \mathbf{u}=0 \\
\rho(\mathbf{u} \cdot \nabla) \mathbf{u}=\nabla \cdot\left[-p \mathbf{I}+\mu\left(\nabla \mathbf{u}+(\nabla \mathbf{u})^{T}\right)\right]+\mathbf{F} \\
\rho C_{p} \mathbf{u} \cdot \nabla T+\nabla \cdot \mathbf{q}=\mathbf{Q}
\end{gathered}
$$

where, $\mathbf{q}=-k \nabla T$

Based on the mass flow rate and the heat exchanger configuration, the Shear Stress Transport (SST) model
Table 2. Design parameters and operating conditions for a single unit of the exchanger.

\begin{tabular}{|l|l|}
\hline Initial & Condition \\
\hline Gas domain & $\mathbf{u}=0 ; p=0 ; k=\left(\frac{10 \cdot \mu}{\rho\left(0.1 \cdot l_{\text {ref }}\right)}\right)^{2} ; \varepsilon=\frac{C_{\mu} k_{\text {init }}^{3 / 2}}{0.1 \cdot l_{\text {ref }}} ; \omega_{\text {init }}=\frac{\sqrt{k_{\text {init }}}}{0.1 \cdot l_{\text {ref }}}$ \\
\hline All domains & $T=298.15 \mathrm{~K}$ \\
\hline Boundary & Condition \\
\hline Inlet & $T=T_{i n} ; u=0, v=-u_{i n}, w=0$ \\
\hline Wall & $\mathbf{u} \cdot \mathbf{n}=0 ; \nabla k_{e} \cdot \mathbf{n}=0 ; \varepsilon=\frac{C_{\mu}^{3 / 4} k_{e}^{3 / 2}}{\kappa_{v} \delta_{w}}$ \\
\hline $\begin{array}{l}\text { Inner tube } \\
\text { wall }\end{array}$ & $T=T_{w}$ \\
\hline Outlet & $p=p_{\text {out }},-\mathbf{n} \cdot \mathbf{q}=0 ; \nabla k_{e} \cdot \mathbf{n}=0 ; \nabla \varepsilon \cdot \mathbf{n}=0$ \\
\hline Symmetry & $\mathbf{u} \cdot \mathbf{n}=0 ;-\mathbf{n} \cdot \mathbf{q}=0 ; \nabla k_{e} \cdot \mathbf{n}=0 ; \nabla \varepsilon \cdot \mathbf{n}=0$ \\
\hline
\end{tabular}

is adopted. The governing equations of two-equation SST model are formulated in terms of $k$ and $\omega$ as (Mentor, 1994)-

$$
\begin{aligned}
\rho \frac{\partial k}{\partial t}+\rho \mathbf{u} \cdot \nabla k= & P-\rho \beta_{o} k \omega+\nabla \cdot\left(\left(\mu+\sigma_{k} \mu_{T}\right) \nabla k\right) \\
\rho \frac{\partial \omega}{\partial t}+\rho \mathbf{u} \cdot \nabla \omega= & \frac{\rho \gamma}{\mu_{T}} P-\rho \beta \omega^{2}+\nabla \cdot\left(\left(\mu+\sigma_{\omega} \mu_{T}\right) \nabla \omega\right)+ \\
& 2\left(1-f_{v 1}\right) \frac{\rho \sigma_{\omega 2}}{\omega} \nabla \omega \cdot \nabla k
\end{aligned}
$$

The default model parameters used to solve the governing equations are defined in the Appendix. Table 2 lists initial conditions for a steady state simulation (Mentor et al., 2003) and boundary conditions used to solve the computational model and achieving preliminary results numerically.

\section{Model validation}

\subsection{Experimental validation}

The validation of numerical results is performed using the experimentally developed correlations by Chen et al. (2014) and a comparison is shown in Figure 5.

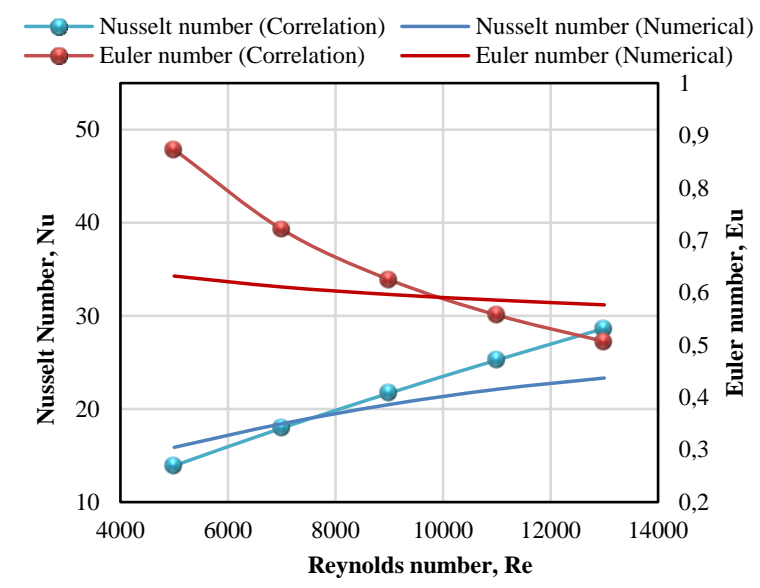

Figure 5. Computational geometry used in the present investigation. 
Correlations for the Nusselt number and Euler number given by (7) and (8) are valid for Reynolds number range of 5000-18000 with a relative error of $2.79 \%$ and $3.70 \%$, respectively. The average percent deviation of numerically predicted Nusselt and Euler numbers from the correlation values is calculated to be $5.61 \%$ and $5.72 \%$, respectively. The deviation accounts for the assumptions in the present study or and the experimental errors in developing the correlations. These deviations are in acceptable range and hence, the results are assumed accurate enough to predict the physical behavior.

$$
\begin{array}{r}
N u=0.053 \operatorname{Re}^{0.756}\left(\frac{D_{o}}{p_{f}}\right)^{-0.212}\left(\frac{L_{f}}{p_{f}}\right)^{-0.294}\left(\frac{W_{f}}{p_{f}}\right)^{0.155} \\
E u=19.14 \operatorname{Re}^{-0.57}\left(\frac{L_{f}}{D_{o}}\right)^{1.32}
\end{array}
$$

\subsection{Mesh independence test}

Mesh independence test is made on the reference fin using temperature difference across the gas domain as an objective property. Five different meshes with 375860, 657449, 997272, 1716992 and 2130500 elements are used in the simulation. The test result suggests the mesh with 1716992 elements as a good choice in regards with the accuracy and computational time.

\section{Results and discussion}

The results predicted from the present study are discussed in this section. Table 3 expresses the dimensionless parameters used to evaluate the performance of the heat exchanger design. The Nusselt number and Euler number are used to assess the heat transfer and pressure loss characteristics of the heat exchanger with different fin geometric profile. As observed in Figure 6, the Nusselt number increases with the Reynolds number which shows thermal performance increases as the flow velocity increases. Moreover, the Nusselt number is higher for $\alpha=1.0$, i.e., for rectangular

Table 3. Performance parameters.

\begin{tabular}{|l|l|}
\hline Performance parameter & Expression \\
\hline Nusselt number, $N u$ & $\frac{h D_{o}}{k}$ \\
\hline Euler number, $E u$ & $\frac{\Delta p}{\frac{1}{2} \rho_{g} u_{\max }^{2}}$ \\
\hline Efficiency index, $\eta$ & $\frac{N u}{\left(\frac{\Delta p}{1 / 2 \rho_{g} u_{i n}^{2}}\right)}$ \\
\hline Area-goodness factor, $j / f$ & $\frac{\left(\frac{N u}{\operatorname{RePr} r^{1 / 3}}\right)}{\left(\frac{\Delta p D_{o}}{1 / 2 \rho_{g} u_{i n}^{2} L_{g}}\right)}$ \\
\hline
\end{tabular}

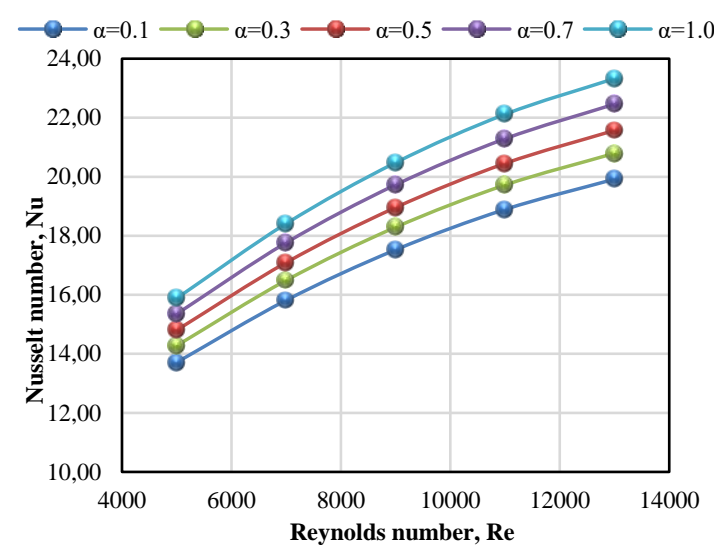

Figure 6. Variation of the Nusselt number with respect to Reynolds number.

fin profile and then decreases as $\alpha$ approaches to 0.1 where the fin geometric profile becomes nearly triangular. This effect results from the decreasing flow velocity as $\alpha$ varies from 1.0 to 0.1 , which further decreases the convective heat transfer. Variation in Euler number with Reynolds number for different fin geometric profiles can be seen from Figure 7. Euler number decreases as $\alpha$ varies from 1.0 to 0.1 , which is a clear demonstration of reduced pressure loss on a transition of rectangular fin profile $(\alpha=1.0)$ to triangular profile $(\alpha=0.1)$. At $\operatorname{Re}=13000$, Euler number for $\alpha=0.1$ decreases by $7.23 \%$ than $\alpha=1.0$.

In order to evaluate the overall performance of the heat exchanger in terms of both, heat transfer and pressure loss, efficiency index (Table III) is calculated. Figure 8 shows a variation in efficiency index with respect to Reynolds number for different fin geometric profiles. The efficiency index increases with the Reynolds number and so thus the overall performance of the heat exchanger design. As observed, efficiency index increases as $\alpha$ goes down from 1.0 to 0.1 . which dictates that the fin with tapered geometric profile performs better in comparison to the conventional rectangular fin geometric profile.

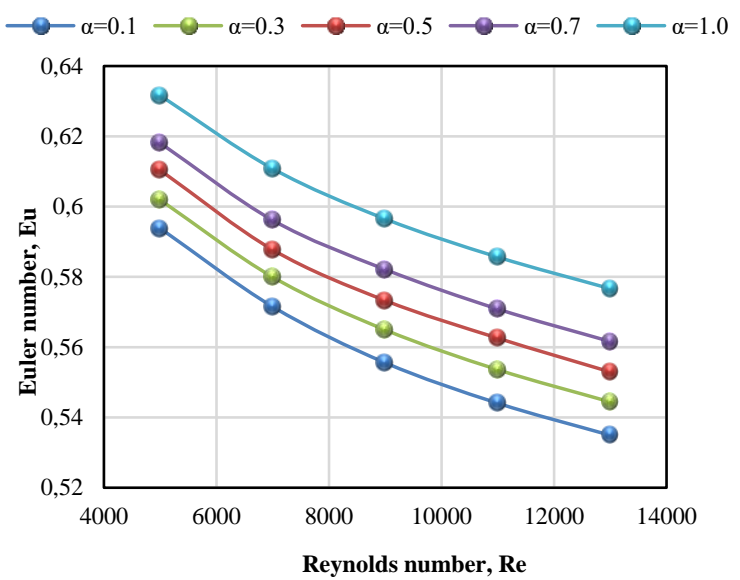

Figure 7. Variation of the Euler number with respect to Reynolds number. 


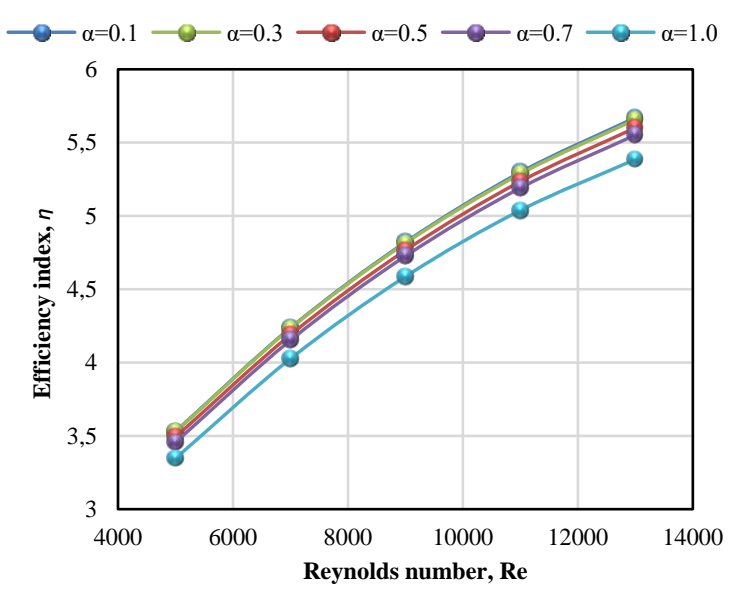

Figure 8. Variation of the efficiency number with respect to Reynolds number.

This trend of efficiency index explains that as $\alpha$ varies from 1.0 to 0.1 , the pressure reduction is dominant than that of increment in thermal performance. In addition, the heat exchanger with $\alpha=0.1$ and $\alpha=0.3$ shows nearly equivalent performance. For instance, at $R e=13000$ efficiency index at $\alpha=0.1$ is only $0.23 \%$ higher than $\alpha=0.3$. Heat transfer through the fin can be predicted from the temperature gradients on the fin surface.

Figure 9 shows temperature gradients on the fin surface of different geometric profiles. The dissipation of the heat from hot gas to the fin is evident from the higher temperatures away from fin and tube interface where the heat is conducted from the fin to the tube wall resulting in lower temperatures in those regions. Relatively, higher temperature gradients on the fin surface with $\alpha=0.1$ are evident of lower heat transfer rate due to the lower temperature difference between the gas
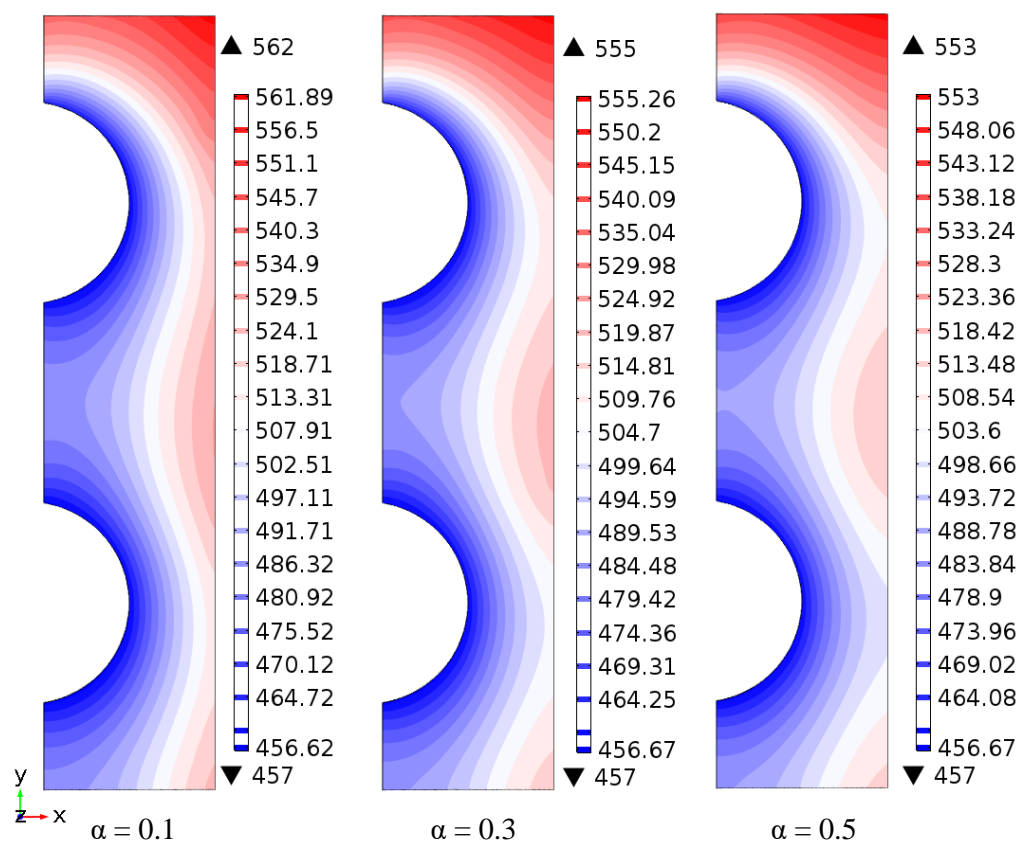

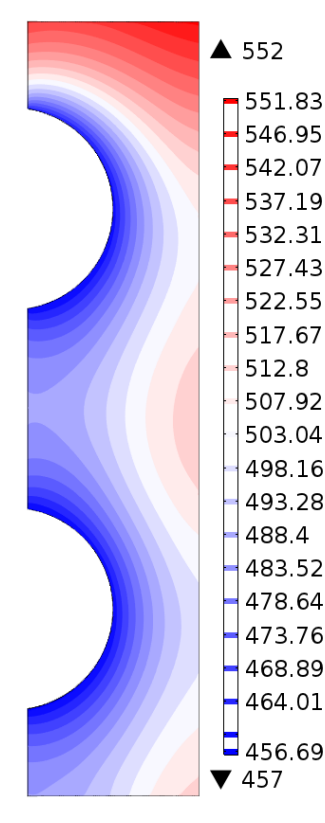

$\alpha=0.7$

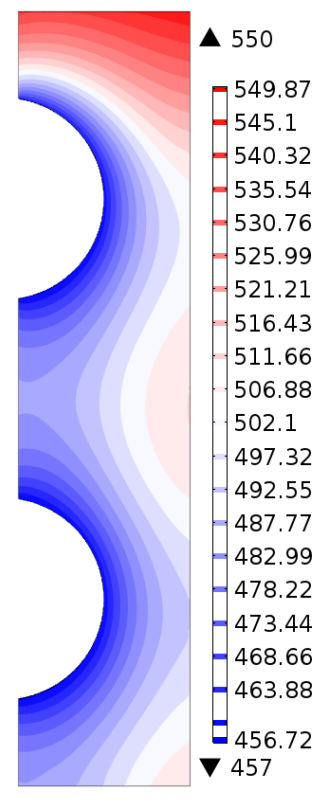

$\alpha=1.0$

Figure 9. Temperature gradients on the fin surface of different geometric profiles. 


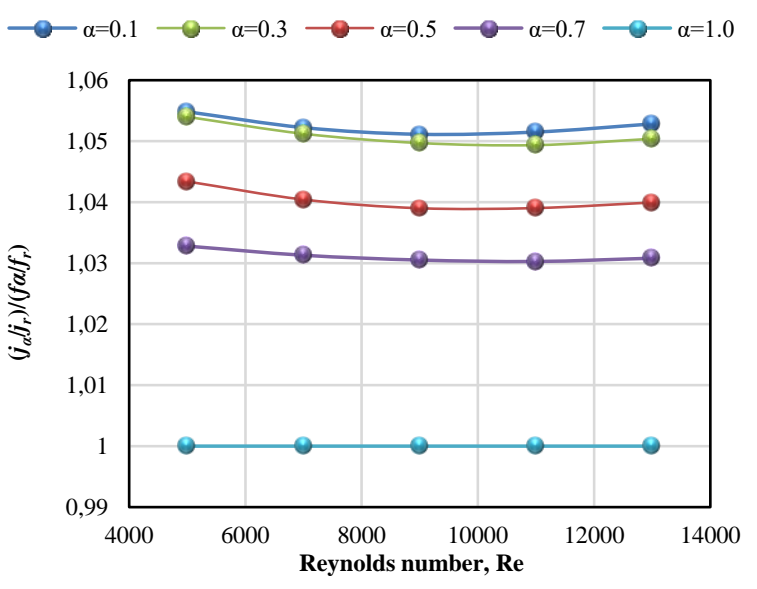

Figure 10. Performance comparison of different geometric fin profile.

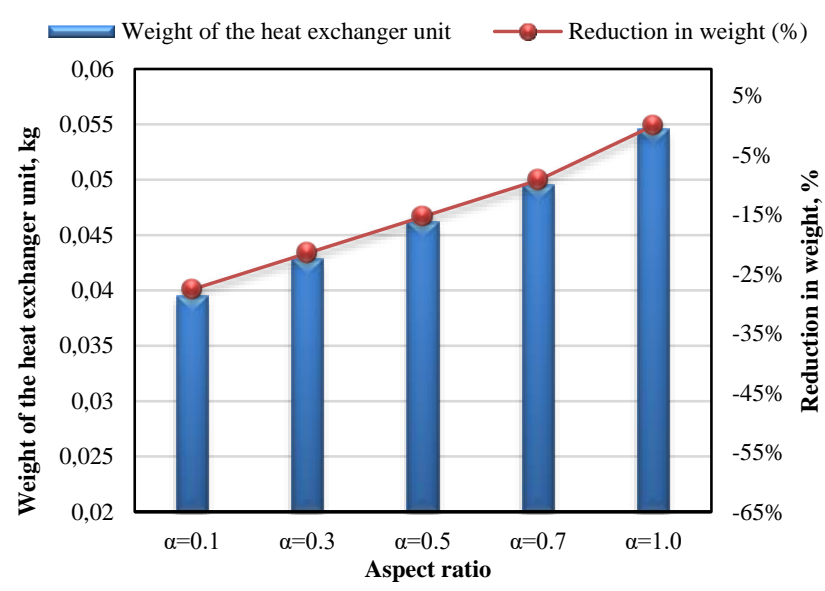

Figure 11. Comparison of the change in weight of heat exchanger unit with different geometric fin profile.

\section{Conclusions}

In the present study, the impact of different fin geometric profiles on the heat transfer performance and pressure loss in a fin and tube heat exchanger design are analyzed. The numerical study concludes that the fin with triangular at $\alpha=0.1$ profile can enhance the heat transfer with reduced pressure loss in comparison to the conventional rectangular fin profile, $\alpha=1.0$. Furthermore, the fin with $\alpha=0.1$ reduces the heat exchanger weight up to $28 \%$ which is always desirable in the industrial applications of fin and tube heat exchangers. The work presented in this paper encourages the further investigation of different possible fin geometric profiles to optimize the material and manufacturing cost which are the main controlling factors in designing a fin and tube heat exchangers at the industrial scale.

\section{Acknowledgements}

This work is a part of the research project: THERMCYC- Advanced thermodynamic cycles utilising low-temperature heat sources (Project No. 1305-0036B).

\section{Nomenclature}

Symbols

$D \quad$ diameter of the tube, $\mathrm{m}$

$E u \quad$ Euler number

F body force vector, $\mathrm{N} / \mathrm{m}^{3}$

$h$ Convective heat transfer coefficient, $\mathrm{W} / \mathrm{m}^{2} . \mathrm{K}$

$K$ thermal conductivity, W/m.K

$k$ turbulent kinetic energy, $\mathrm{m}^{2} / \mathrm{s}^{2}$

$L$ length, $\mathrm{m}$

$\mathrm{Nu}$ Nusselt Number

$p$ pressure, $\mathrm{Pa}$

$\Delta p$ pressure difference across the gas domain, $\mathrm{Pa}$

$\operatorname{Pr}$ Prandtl number

Q heat flux vector, $\mathrm{W} / \mathrm{m}^{2}$

Re Reynolds number

$T$ temperature, $\mathrm{K}$

$Q$ heat source or sink, $\mathrm{W} / \mathrm{m}^{3}$

$u$ flow velocity, $\mathrm{m} / \mathrm{s}$

u average velocity vector, $\mathrm{m} / \mathrm{s}$

$\omega \quad$ Specific dissipation rate, $1 / \mathrm{s}$

$\rho$ density, $\mathrm{kg} / \mathrm{m}^{3}$

$\mu \quad$ dynamic viscosity of the gas, Pa.s

\section{Subscripts}

$g \quad$ gas or gas domain

$l \quad$ liquid

$f \quad$ fin

$w$ inner tube wall

$i$ inner tube

$o$ outer tube

max maximum

$r$ reference fin geometric profile

\section{References}

Y. A. Cengel, J. M. Cimbala, and R. H. Turner. Fundamentals of thermal-fluid sciences, Fourth edition in SI units. McGraw-Hill. 2012.

H. T. Chen, and J. R. Lai. Study of heat-transfer characteristics on the fin of two-row plate finned-tube heat exchangers. Int. J. Heat Mass Tran., 55: 4088-4095, 2012. doi:10.1016/j.ijheatmasstransfer.2012.03.050.

H. Chen, Y. Wang, Q. Zhao, H. Ma, Y. Li, and Z. Chen. Experimental Investigation of Heat Transfer and Pressure Drop Characteristics of H-type Finned Tube Banks. Energies, 7(11): 7094-7104, 2014. doi:10.3390/en 7117094.

Y. Jin, G. H. Tang, Y. L. He, and W. Q. Tao. Parametric study and field synergy principle analysis of H-type finned tube bank with 10 rows. Int. J. Heat Mass. Tran., 60: 241-251, 2013. doi: 10.1016/j.ijheatmasstransfer.2012.11.043.

F. R. Menter. Two-Equation Eddy-Viscosity Turbulence Models for Engineering Applications. AIAA Journal, 38(8): 1598-1605, 1994. doi:10.2514/3.12149.

F. R. Menter, M. Kuntz, and R. Langtry. Ten years of industrial experience with the SST Turbulence Model. Turbulence Heat and Mass Transfer, 2003. doi: 10.1.1.460.2814.

L. Tong. 3D numerical analysis of heat transfer characteristics for H-type finned tube. Mech. Electr. Eng. Mag., 152: 79$81,2007$.

Y. C. Wang, and G. H. Tang. Acid condensation and heat transfer characteristics on H-type fin surface with bleeding 
dimples and longitudinal vortex generators. Chin. Sci. Bull., 59(33): 4405-4417, 2014. doi:10.1007/s11434-0140564-3.

X. Yu, Y. Yuan, Y. Ma, and H. Liu. Experimental tests and numerical simulation on heat transfer and resistance characteristics of H-type finned tube banks. J. Power Eng., 30: 433-438, 2010. doi:10.1016/j.egypro.2017.03. 1014.

Z. Zhang, Y. Wang, and Q. Zhao. Numerical study on performance optimization of H-type finned tubes. $J$. Power Eng., 30: 941-946, 2010.

X. B. Zhao, G. H. Tang, X. W. Ma, Y. Jin, and W. Q. Tao. Numerical investigation of heat transfer and erosion characteristics for H-type finned oval tube with longitudinal vortex generators and dimples. Appl. Energy, 127: 93-104, 2014. doi:10.1016/j.apenergy.2014.04.033.

\section{Appendix}

In (5), $P=\min \left(P_{k}, 10 \rho \beta_{o} k \omega\right)$

where, $P_{k}=\mu_{T}\left(\nabla \mathbf{u}:\left(\nabla \mathbf{u}+(\nabla \mathbf{u})^{T}\right)-\frac{2}{3}(\nabla \cdot \mathbf{u})^{2}-\frac{2}{3} \rho k \nabla \cdot \mathbf{u}\right.$

The turbulent viscosity is, $\mu_{T}=\frac{\rho a_{1} k}{\max \left(a_{1} \omega, S f_{v 2}\right)}$

where, $\mathrm{S}$ is the characteristic magnitude of the mean velocity gradients,

$S=\sqrt{2 S_{i j} S_{i j}}$

The other model constants are given in terms of interpolation functions as,

$\phi=f_{v 1} \phi_{1}+\left(1-f_{v 1}\right) \phi_{2}$ for $\phi=\beta, \gamma, \sigma_{k}, \sigma_{\omega}$ and $f_{v 1}=\tanh \left(\theta_{1}^{4}\right)$

$\theta_{1}=\min \left[\max \left(\frac{\sqrt{k}}{\beta_{o} \omega l_{\omega}}, \frac{500 \mu}{\rho \omega l_{\omega}^{2}}\right), \frac{4 \rho \sigma_{\omega 2} k}{C D_{k \omega} l_{\omega}^{2}}\right]$

where, $1 \mathrm{w}$ is the distance to the closest wall.

$C D_{k \omega}=\max \left(\frac{2 \rho \sigma_{\omega 2}}{\omega} \nabla \omega \cdot \nabla k, 10^{-10}\right)$
$f_{v 2}=\tanh \left(\theta_{2}^{2}\right)$ and $\theta_{2}=\max \left(\frac{2 \sqrt{k}}{\beta_{o} \omega l_{\omega}}, \frac{500 \mu}{\rho \omega l_{\omega}^{2}}\right)$

The other default model parameter values are,

$\beta_{1}=0.075, \gamma_{1}=5 / 9, \sigma_{k 1}=0.85, \sigma_{\omega 1}=0.5$,

$\beta_{2}=0.0828, \gamma_{2}=0.44, \sigma_{k 2}=1.0, \sigma_{\omega 2}=0.856$,

$\beta_{o}=0.09, a_{1}=0.31$

The Reynolds number is calculated as:

$R e=\frac{\rho u_{\max } D_{o}}{\mu}$

The gas-side convective heat transfer coefficient is determined by overall heat transfer coefficient as:

$\frac{1}{U}=\frac{1}{h_{g}}+\frac{A_{t}}{A_{i}}\left(\frac{1}{h_{l}}+\frac{\left(D_{o}-D_{i}\right)}{2 K}\right)$

On further simplification,

$\frac{1}{U A_{t}}=\frac{1}{h_{g} A_{t}}+\frac{1}{h_{l} A_{i}}+\frac{1}{\left[\frac{2 K A_{i}}{\left(D_{o}-D_{i}\right)}\right]}$

Since the heat transfer coefficient inside the tube is high $\left(\sim 10^{4}\right.$ $\left.\mathrm{W} / \mathrm{m}^{2} . \mathrm{K}\right)$, the second term in Eq. (x) is omitted. The above equation can be further simplified without losing accuracy as the tubes being analyzed are of small thickness $\left(\sim 10^{-3} \mathrm{~m}\right)$ and higher thermal conductivity $(\sim 50 \mathrm{~W} / \mathrm{m} . \mathrm{K})$ which makes the third term very small and hence negligible. This results in a much more straightforward expression,

$$
\begin{aligned}
& \frac{1}{U A_{t}}=\frac{1}{h_{g} A_{t}} \\
& U=h_{g}
\end{aligned}
$$

Overall heat transfer coefficient can be determined as:

$$
U=\frac{Q_{t}}{A_{t} \Delta T_{l m}}
$$

where, $\Delta T_{l m}=\frac{\left(T_{w}-\bar{T}_{\text {in }}\right)-\left(T_{w}-\bar{T}_{\text {out }}\right)}{\ln \left[\frac{\left(T_{w}-\bar{T}_{\text {in }}\right)}{\left(T_{w}-\bar{T}_{\text {out }}\right)}\right]}$ 